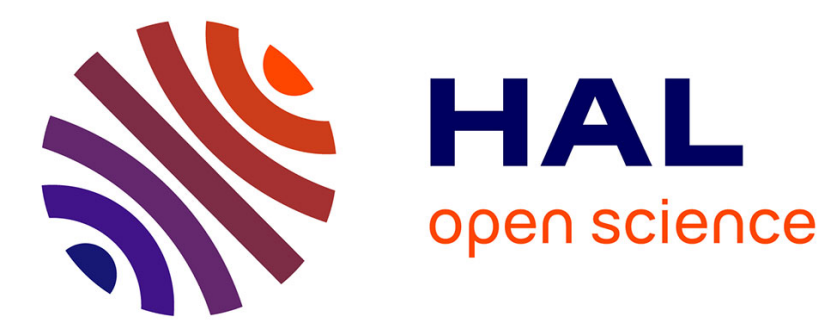

\title{
Measurement of the natural frequencies and critical speed of roll-tensionned discs
}

\author{
E. Aubry, D. Fendeleur, F.M. Schmitt, M. Renner
}

\section{To cite this version:}

E. Aubry, D. Fendeleur, F.M. Schmitt, M. Renner. Measurement of the natural frequencies and critical speed of roll-tensionned discs. Experimental Techniques, 2000, 24 (4), pp.22-25. 10.1111/j.17471567.2000.tb00921.x . hal-00956809

\section{HAL Id: hal-00956809 \\ https://hal.science/hal-00956809}

Submitted on 7 Mar 2014

HAL is a multi-disciplinary open access archive for the deposit and dissemination of scientific research documents, whether they are published or not. The documents may come from teaching and research institutions in France or abroad, or from public or private research centers.
L'archive ouverte pluridisciplinaire HAL, est destinée au dépôt et à la diffusion de documents scientifiques de niveau recherche, publiés ou non, émanant des établissements d'enseignement et de recherche français ou étrangers, des laboratoires publics ou privés. 


\title{
Measurement of the natural frequencies and critical speed of
}

\section{roll-tensioned discs.}

\author{
E.Aubry - D.Fendeleur - F.M.Schmitt - M.Renner
}

ESSAIM (Ecole Supérieure des Sciences Appliquées pour l'Ingénieur de Mulhouse)

12 rue des Frères Lumière

68093 Mulhouse cedex - France

Tel : (33) 389336943 - Fax : (33) 389423282

email : e.aubry@essaim.univ-mulhouse.fr - dfendel@club-internet.fr - f.m.schmitt@essaim.univ-mulhouse.frm.renner@univ-mulhouse.fr

Thin steel discs can be used to make circular saw plates. The process of roll-tensioning is traditionally used to increase the stability of rotating discs at high rotation speed, and so dynamic properties and critical speed, which determines the maximum stable rotation speed of the disc. Rolltensioning consists in introducing a residual stress state in the plate. The structure is locally deformed between two opposite pinch rollers in rotation which rotate the plate. This creates a plastic deformation along a circular zone at constant radius. Manufacturers are generally able to roll tension acceptably through know-how gained from experience. However, this is done, very often, without any scientific knowledge. In parallel, many efficient theories have been developed by several scientists to explain the dynamic phenomena which occur. Our principal target in this paper is to expose how we developed a reliable method to determine the critical speed in connection with the roll-tensioning process applied to the structure. This method is to allow the quantification of roll tensioning during the production process, in industrial situation.

To perform our tests, we adhered as closely as possible to the normal working conditions of the type of tool : a circular rotating tool, with a bore, fixed on an arbor with flanges of a given diameter. We used modal analysis methods to evaluate the natural frequencies of each mode shape when the disc is not rotating . Then their evolution with the increase of the rotation speed were followed. Experimental results and theoretical results described in the bibliography were compared. 
This article is composed of three parts. The first part describes the experimental bench used for the

tests. It presents also the software developed for the determination of the modal properties of the plate. In the second part, we show that the results obtained for a disc which has not been roll-tensioned and the theoretical values are comparable. This proves the effectiveness of our method. In the third part, by evaluating the natural frequencies and the critical speed of several discs roll-tensioned differently, we clearly point out the influence of the roll-tensioning process on the dynamic properties of the structure.

Then, we conclude with a description of the advantages and the limits of our method.

\section{Description of the test stand :}

The test stand (Figure 1) is composed of the different parts described below :

- a D.C. motor with a speed variator allows any rotation speed between 0 and $3000 \mathrm{rpm}$,

- a pair of flanges (outside diameter $=70 \mathrm{~mm}$ ) to fix the disc,

- an eddy current proximity system to measure the vibrations,

- an impact hammer fitted with a force sensor to excite and measure the excitation force),

- a signal analyzer to estimate the frequency response function of the structure,

- a computer with $\operatorname{Matlab}^{\circledR}$ software to extract the data, to propose an interpretation of the measure and to plot the Campbel's diagram.

All the equipment described assists in finding the natural modes when the structure is running or not by the use of the classical modal analysis methods. In each case, the impact hammer, fitted with a force sensor, provides the excitation and the response is measured by the proximity sensor. The sensor measures the lateral displacements at the rim of the plate, where the vibration have the largest amplitudes. The spectrum analyzer stores the time signal then proceeds to the signal analysis (windowing, averaging, FFT, etc...) and calculates the Frequency Response Function (real and imaginary parts, phase and 
magnitude). Then, a Matlab ${ }^{\circledR}$ script has been written to perform a post-analysis and to display the resulting curves.

\section{Natural frequencies and critical speed of a rotating disc without tensioning :}

When a steel disc is not rotating, the natural frequencies of this disc clamped at the bore and free at

the rim are given by Blevins ${ }^{1}: \quad \quad \mathrm{f}_{\mathrm{ij}}=\frac{\lambda^{2}{ }_{\mathrm{ij}}}{2 \pi \mathrm{a}^{2}}\left(\frac{\mathrm{Eh}^{2}}{12 \rho\left(1-v^{2}\right)}\right)^{1 / 2}$

where $\mathrm{a}$ is the outside diameter, $\mathrm{h}$ the thickness, $\mathrm{E}$ the elasticity module of the material, $\rho$ the specific mass and $v$ the Poisson's coefficient. The boundary conditions allow to calculate the coefficient $\lambda_{i j}$ by imposing given relations on the bending moments equation and on shearing stresses equations ${ }^{1,2} . \lambda_{i j}$ is related to the ratio of the diameters (outside diameter / clamping diameter). The mode shapes for each natural frequency are characterized by some nodal diameter and/or by some nodal circles. The number of nodal diameter is indicated by the letter $i$, and the number of nodal circle is $j$.

When the disc is in motion, some rotation stresses are induced by the centrifugal forces $3,4,5$.

These modify the natural frequencies of the disc according to the equation :

$$
\mathrm{f}_{\mathrm{s}}^{2}=\mathrm{f}^{2}+\mathrm{K}\left(\frac{\Omega}{60}\right)^{2}
$$

where $\mathrm{f}$ is the natural frequency calculated as indicated in Eq.1, $\Omega$ the rotation speed in $\mathrm{rpm}$ and $\mathrm{K}$ dimensionless value.

The phenomenon of stationary waves is represented for a given natural frequency by a mode shape. This is due to the existence of two different waves around the disc, with the same frequency but travelling with the rotation speed in two opposite directions. When the disc rotates, the rotation speed is added to the propagation speed of the travelling wave in the direction of rotation (forward waves) and, is substracted from the propagation speed of the travelling wave in the opposite direction (backwards waves). This leads to a splitting of each mode shape into two different frequencies. Only mode shapes 
with certain nodal diameters are affected. Therefore, the frequencies of the forward modes increase and the frequencies of the backward modes decrease in relation to the rotation speed ${ }^{6}$ :

$$
\mathrm{f}_{\mathrm{f}}=\mathrm{f}_{\mathrm{s}}+\mathrm{i} \frac{\Omega}{60} \text { and } \mathrm{f}_{\mathrm{b}}=\mathrm{f}_{\mathrm{s}}-\mathrm{i} \frac{\Omega}{60}
$$

where $f_{f}$ and $f_{b}$ respectively are the frequencies associated with the forward and the backward waves; $i$ is the number of nodal diameter.

We measured the mode splitting phenomenon. The disc used was not roll-tensioned, had an outside diameter of $350 \mathrm{~mm}$, a thickness of 2,6 $\mathrm{mm}$ and the flanges had a diameter of $70 \mathrm{~mm}$. The natural frequency shown on the figure 2 was $470 \mathrm{~Hz}$ when the disc was stopped. Through a modal analysis, it was determined that the mode shape with four nodal diameters $(i=4)$.

The split modes exist for all the natural frequencies. A theoretical special rotation speed where the backward frequency tends toward zero can be found. This leads to the determination of the critical speed which is «the first rotation speed for a backward frequency reaching zero» 6, 7, 8, 9 . A special representation is used to visualize the evolution of each frequency depending on the rotation speed : it is called the Campbell's diagram, which can be theoretically calculated too ${ }^{6}$.

For the measurement, we intentionally divided the frequency range into two parts to have a better accuracy. The figure 3a shows both the results theoretical and experimental on a range of 50 to $250 \mathrm{~Hz}$ and the figure $3 \mathrm{~b}$ both the results theoretical and experimental on a range of 200 to $1000 \mathrm{~Hz}$. On the fist range, the three first modes can be isolated. In addition, the unbalance due to the run-out defect of the disc disturb the measurement and affects the FRF. It is more difficult to extract the interesting values into the FRFs' signal. But, by comparison with the theoretical simulation, the splitting of the second mode is clearly visible. On the higher range of frequency (200 to $1000 \mathrm{~Hz})$, the signal are less disturbed and the measure can more easily used for a post-treatment. In this case, the relation theory-experimentation is obvious. The critical speed can be found by extrapolating the experimental curves. The first frequency 
which tends towards zero corresponds to the mode with two nodal diameters. The critical speed is about $4000 \mathrm{rpm}$.

\section{Natural frequencies and critical speed for a roll-tensioned idling disc :}

The roll-tensioning process significantly modifies the natural properties of the disc, whether it is stopped or rotating. For several discs rolled with different pressure applied to each, the natural frequencies were measured. The results given in the chart (Tab.1) are classified by increasing pressure. The natural frequencies for the clamped disc change with the increased application of pressure. A specific evolution can be found (Fig.4). Other tests performed on several discs with various dimensions confirm these trends. Some modelings cited in the bibliography $6,8,9,10$ confirm this too.

When a tensioned disc rotates, the global state of stresses inside the disc is a combination of the residual stresses coming from the tensioning, of the centrifugal stresses and of the thermal stresses. This modifies the dynamic behavior of the plate and changes the critical speed. Actually, roll-tensioning is done on purpose, in order to improve the stability of the rotating disc.

The equation of motion taking into account these phenomena has been solved in numeric form

years ago by Shajer ${ }^{11,12}$. Because of the number of tensioning methods and because of the difficulty to check the quality of the tension, it is very hard to use the theoretical simulation to qualify the tensioning and to estimate the critical speed.

After several measurements, the Campbell's diagrams were established for the five previous discs. In this article, the evolution of the backward and the forward frequencies are shown Fig.5 for two given modes (mode shape involving two and four nodal diameters). The backward and forward frequencies are systematically in a special order : B5 - B99 - B4 - B6 - B8. B5 is less tensioned than B99, B99 is less tensioned than B4, etc... An example can be given : the B8 disc has his natural frequencies always over the B5's frequencies for every rotation speed. This means that B8 has a higher critical speed than B5. 
Actually, it shows the benefit of the roll-tensioning process on the stability of a rotating disc. The curves on the Campbell's diagram were interpolated to give the critical speed for the five discs. For the disc B5, we found the critical speed at $3900 \mathrm{rpm}$. For B99, it is $4000 \mathrm{rpm}$; for B4, $4350 \mathrm{rpm}$; for B6, $4400 \mathrm{rpm}$ and for $\mathrm{B} 8,5150 \mathrm{rpm}$.

\section{Conclusion :}

At first and as expected, we have shown that the roll-tensioning process has an obvious effect to increase the critical speed and consequently the stability of the rotating disc. But, our target was to point out, through the measurements of the dynamic properties, the relation between the amount of the « tension » applied and the positive variation of the critical speed. Also, with the Campbell's diagram, it is possible to determine the critical speed and to guess the tension. This makes a quality control of the rolltensioning process possible.

Although the techniques of dynamic analysis and modal analysis are quite easy to use in the laboratory, it is difficult to apply the method developed in this article, to industry. The complexity of the measurements and the use of the experimental data require very skilled people. Nevertheless, based on our experience, we can imagine a simplest method to make the measurements. Actually, if it is absolutely necessary to determinate the critical speed to appreciate the influence of the tension on the stability of a rotating disc, the effect of the tensioning can be shown by measuring the first natural frequencies of simple clamped disc.

On our test bench, we evaluated the evolution of these first frequencies when the disc is clamped with flanges (the rim is free). The figure 4 shows this particular evolution. To measure the tension, the literature usually proposes to focus on the frequencies related to the modes $\mathrm{f}_{00}$ and $\mathrm{f}_{04}$. Some devices using this method can be found on the market ${ }^{13}$. But in working conditions, we showed that this technique while interesting, has some disadvantages : the reliability of the clamping conditions are poor, the measurement 
the first natural frequency are difficult and the integration in a control process is problematic due to clamping issues. A new method for fixing the disc is in progress in order to overcome these problems.

\section{References :}

1. Blevins R.D. 'Formulas for natural frequency and modes shape' - 1979 - Editions Krieger

2. Vogel S.M. and Skinner D.W. 'Natural frequencies of transversely vibrating uniform annular plates' - Journal of pplied mechanics - 1965 (12) - p.926-931

3. Hackenberg P. - 'Spannungen in mechanisch und thermisch vorgespannten Kreissägenblättern' - 1974 - Aachen

4. Southwell R.V. 'On the free transverse vibrations of an uniform circular disc clamped at its center and on the effect of rotation' - Proceedings of Royal Society London - 1992 - 101 - p.133-153

5. Mote C.D. 'free vibration of initially stressed circular discs' - Journal of engineering for industry - Transactions of the ASME - 1965 - 87 (may) - p.258-264

6. Shajer G.S. 'Simple formulas for natural frequencies and critical speeds of circular saws' - Forest product journal - 1986 36 - p. $37-43$

7. Szymani R. - 'Latest developments in circular saws tensioning' - International Union of Forestry Research Organization 1983 - Madison - Wisconsin - US Products Laboratory

8. Shajer G.S. and Mote C.D. ‘Analysis of optimal roll tensioning for circular saw stability’ - 1984 - p.264-282

9. Szymani R. and Mote C.D.- 'Theoretical and experimental analysis of circular saw tensionning' - Wood sciences Technology - 1979 - 13 - p. 211-237

10. Szymani R. and Mote C.D.- 'Circular saw stiffness as a measure of tension' - Forest product journal - 1977 - 27 -N3 - p. 28-32

11. Shajer G.S. 'Optimizing saw design and operation' - Circular saw natural frequency program SAWFQ2 - 1979

12. Shajer G.S. 'CSAW : Guided circular saw vibration and stability program' - 1988 - Weyerhaueser Technology Center Tacoma

13. Szymani R. 'Used and maintenance of thin circular saws at california Cedar products Company' - Holz als Roh- und Werkstoff’ - 1987 - 45 - p.319-322 


\section{List of figures :}

Fig.1 : Testing bench

Fig. 2 : Forward and Backward waves

Fig. 3a : Campbell's Diagram (B99) - Theory and Measurements - 50 to $250 \mathrm{~Hz}$

Fig. 3b : Campbell's Diagram (B99) - Theory and Measurements - 200 to $1000 \mathrm{~Hz}$

Fig. 4 : Natural frequencies depending on the roll-tensioning amount

Fig. 5: Influence of the roll-tensioning on the modes $\mathrm{f}_{02}$ and $\mathrm{f}_{04}$

Tab.1 : Natural frequencies of roll-tensioned discs 


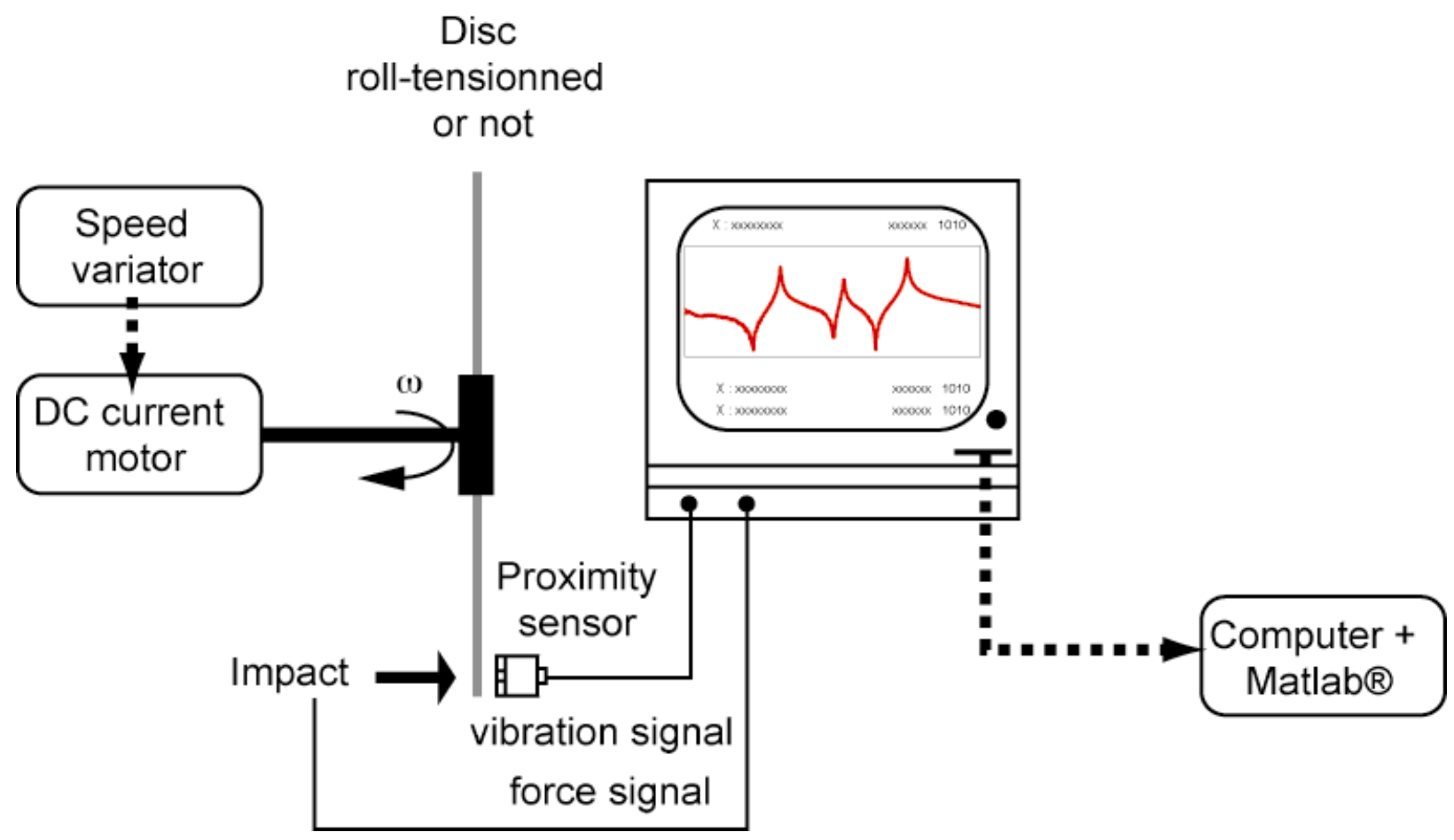

Fig.1 : Testing bench

Aubry/Fendeleur/Schmitt/Renner 


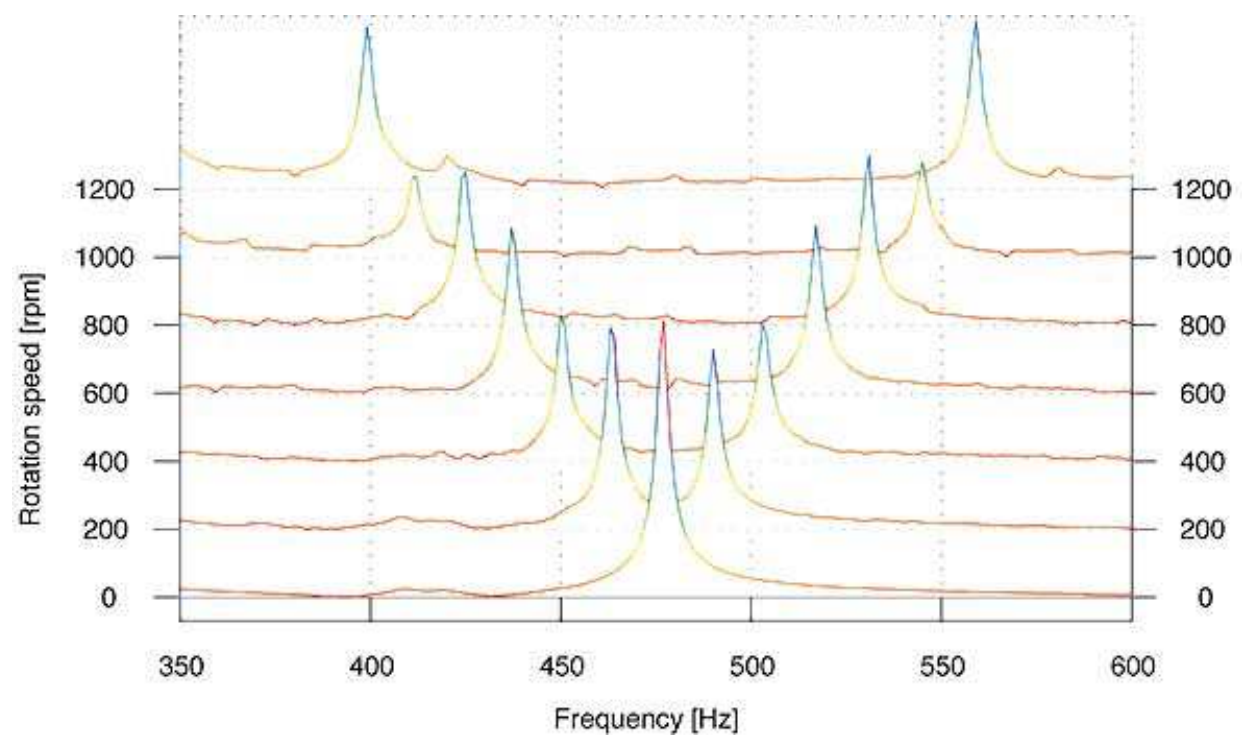

Fig. 2 : Forward and Backward waves 

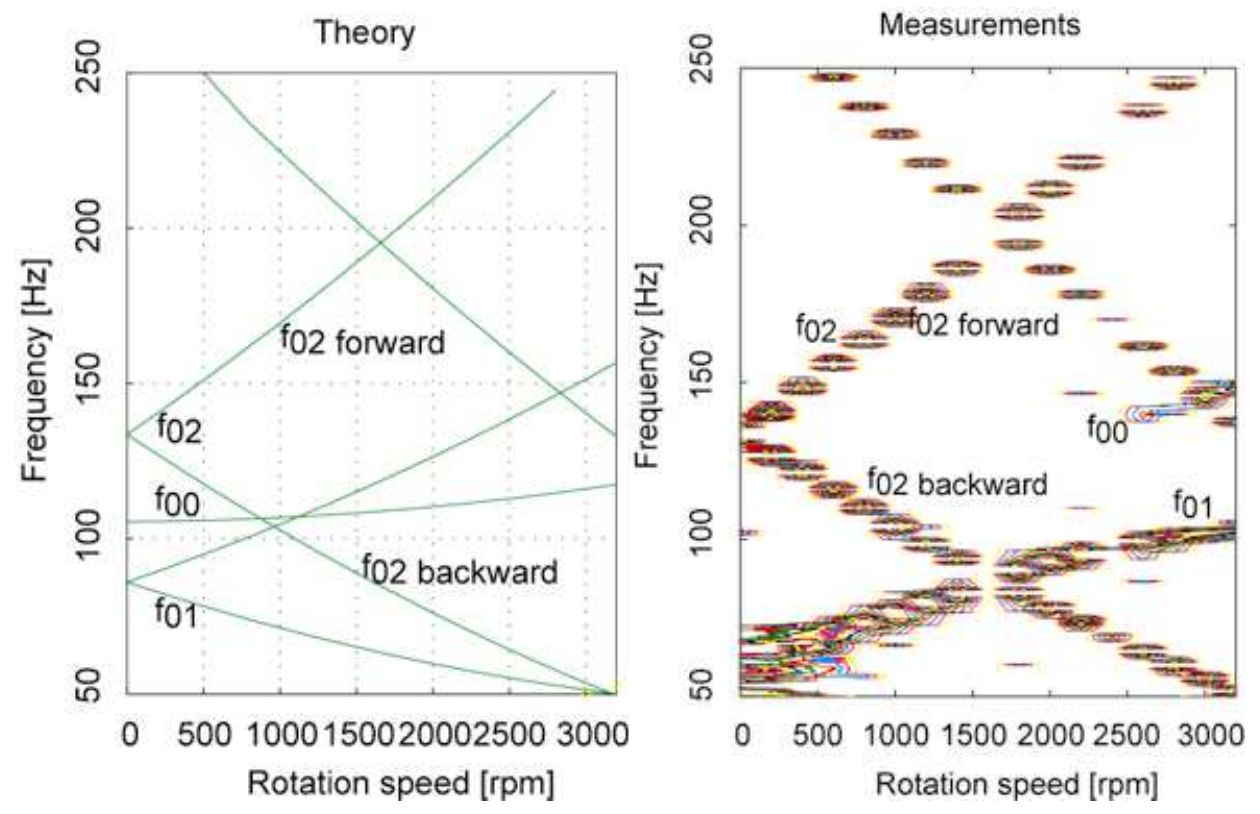

Fig. 3a : Campbell's Diagram (B99) - Theory and Measurements - 50 to $250 \mathrm{~Hz}$

Aubry/Fendeleur/Schmitt/Renner 

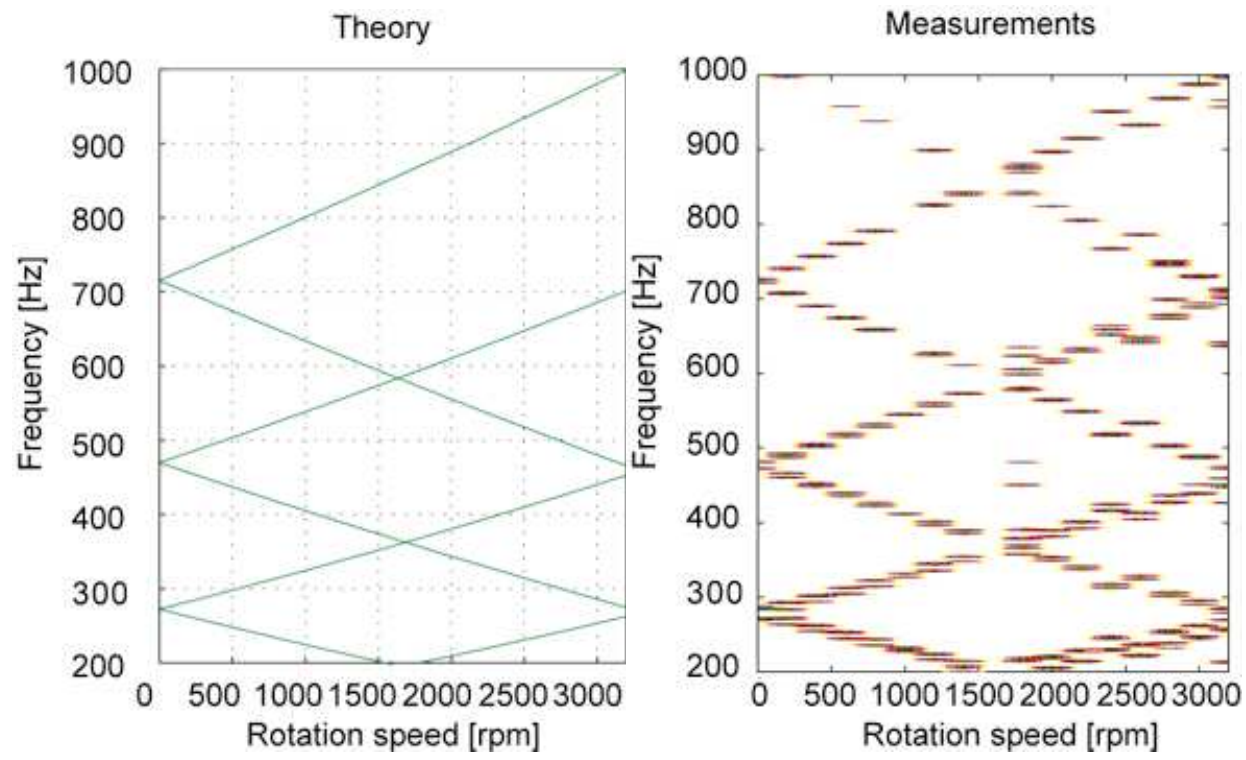

Fig. 3b : Campbell's Diagram (B99) - Theory and Measurements - 200 to $1000 \mathrm{~Hz}$

Aubry/Fendeleur/Schmitt/Renner 


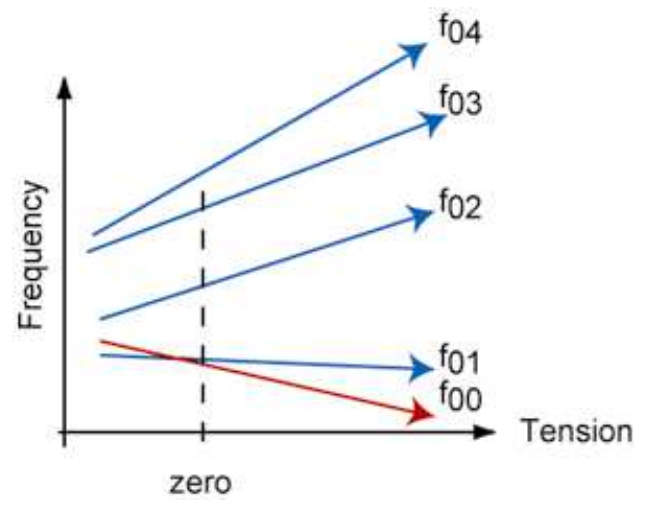

Fig.4 : Natural frequencies depending on the roll-tensioning amount

Aubry/Fendeleur/Schmitt/Renner 

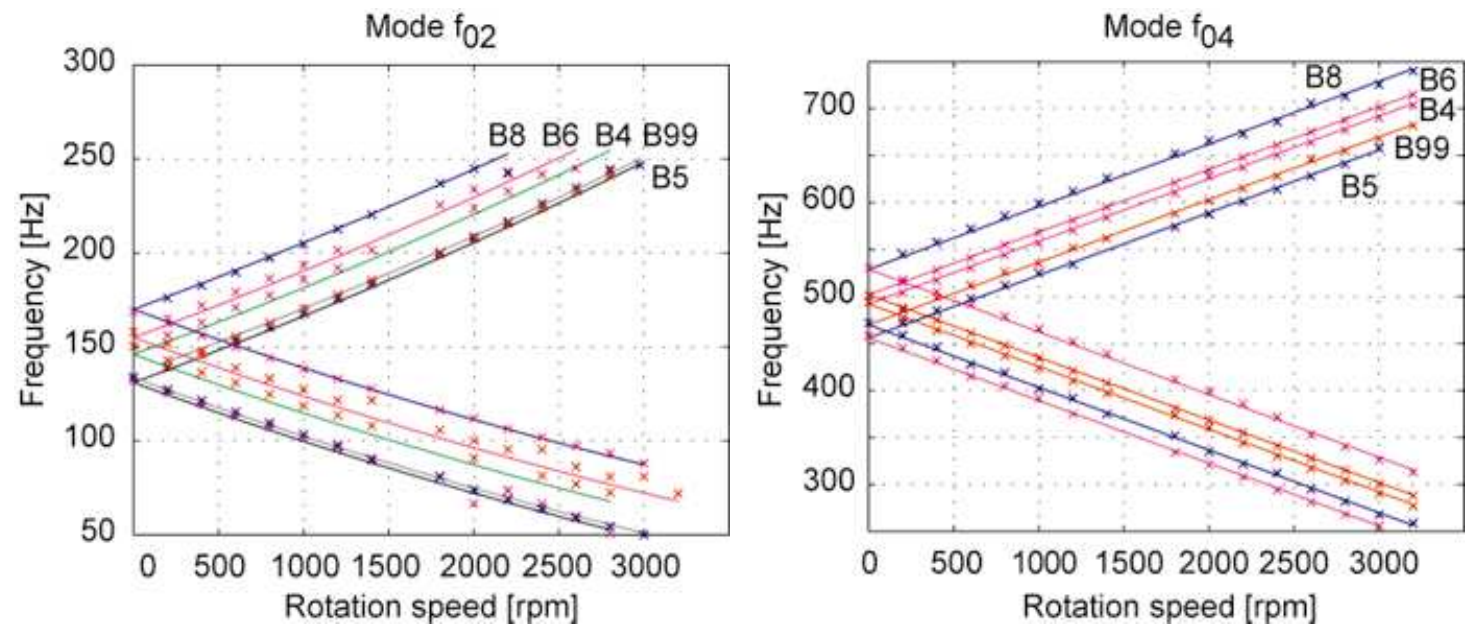

Fig.5: Influence of the roll-tensioning on the modes f02 and f04 


\begin{tabular}{|c|c|c|c|c|c|}
\hline & B5 & B99 & B4 & B6 & B8 \\
\hline $\mathbf{f}_{00}$ & 102 & 105 & 101 & 92 & 78 \\
\hline $\mathbf{f}_{01}$ & 119 & $?$ & 120 & 122 & 119 \\
\hline $\mathbf{f}_{02}$ & 131 & 134 & 146 & 155 & 170 \\
\hline $\mathbf{f}_{03}$ & 264 & 272 & 291 & 301 & 324 \\
\hline $\mathbf{f}_{04}$ & 456 & 470 & 492 & 502 & 529 \\
\hline $\mathbf{f}_{05}$ & 695 & 715 & 741 & 749 & 779 \\
\hline
\end{tabular}

Tab. 1 : Natural frequencies of roll-tensioned discs 